\title{
Testing QCD with Jet Physics at CDF
}

\author{
Eve Kovács \\ The CDF Collaboration \\ Fermi National Accelerator Laboratory \\ P.O. Box 500, Batavia, Illinois 60510
}

August 1994

Proceedings of the Eighth Meeting of the Division of Particles and Fields of the American Physical Society (DPF'94), Albuquerque, NM, August 2-6, 1994. 


\section{Disclaimer}

This report was prepared as an account of work sponsored by an agency of the United States Government. Neither the United States Government nor any agency thereof, nor any of their employees, makes any warranty, express or implied, or assumes any legal liability or responsibility for the accuracy, completeness, or usefulness of any information, apparatus, product, or process disclosed, or represents that its use would not infringe privately owned rights. Reference herein to any specific commercial product, process, or service by trade nane, trademark, manufacturer, or otherwise, does not necessarily constitute or imply its endorsement, recommendation, or favoring by the United States Government or any agency thereof. The views and opinions of authors expressed herein do not necessarily state or reflect those of the United States Government or any agency there of. 
CDF/PUB/JET/PUBLIC/2726

FERMILAB CONF-94/215-E

July 28,1994

\title{
Testing QCD with Jet Physics \\ at CDF
}

\author{
The CDF Collaboration
}

Presented by

Eve Kovács

Fermilab, Box 500, Batavia

IL 60510, U.S.A.

\begin{abstract}
We present a variety of jet cross section measurements in $p \bar{p}$ collisions at $\sqrt{s}=1.8 \mathrm{TeV}$, using $19.5 \mathrm{pb}^{-1}$ of data collected by the Collider Detector at Fermilab during the 1992-93 run of the Fermilab Tevatron. We discuss the impact of these measurements on the status of precision tests of QCD.
\end{abstract}

\section{Introduction}

Jet cross section measurements at CDF can be used to determine fundamental QCD quantities, perform a large variety of QCD tests and place unique constraints on the value of the gluon distribution. The improved precision of the data from the 1992-93 run, combined with the recent advances in the theoretical calculations means that precision tests of QCD at the $\pm 20 \%$ level have become possible. Furthermore, the scope of these tests has been extended to regions of high $|\eta|$, where the boundaries of kinematic phase space can be probed. As a result of these improvements, CDF measurements can now be used in global parton distribution analyses to provide unique constraints on the gluon distributions for $10^{-3} \lesssim x \lesssim .5$

Here, we present preliminary results for the measurements of the inclusive jet cross section, the two-jet differential cross section, and the "same-side" (SS) to "oppositeside" (OS) dijet ratio at $\sqrt{s}=1800 \mathrm{GeV}$. The $19.5 p b^{-1}$ of data were collected by the 
CDF Collaboration during the 1992-93 run of the Fermilab Tevatron Collider. Jets are identified by the CDF jet-cone algorithm, ${ }^{1}$ using a cone of radius $\sqrt{(\Delta \eta)^{2}+(\Delta \phi)^{2}}=$ 0.7 . to measure the transverse energy, $E_{T}$, of a jet.

\section{The Inclusive Jet Cross Section}

The measurement of the inclusive jet cross section provides a stringent test of QCD over an enormous dynamic range. At high $E_{T}$, where the cross section is dominated by quark-quark scattering, deviations from the QCD predictions could signal the presence of quark substructure. At lower $E_{T}$ 's, the cross section is dominated by gluon-gluon scattering, and the measurement provides a leading order (LO) constraint on the gluon distribution. For this analysis, the jets are required lie in the region $0.1<|\eta|<0.7$, and the measured jet $E_{T}$ 's are corrected for detector effects. ${ }^{2}$ The total systematic uncertainty is around $13 \%$ over most of the $E_{T}$ range, whilst the statistical uncertainty is less than $2 \%$ over this range.

In Figs. 1a) and 1b), we show the inclusive jet spectrum on a logarithmic and linear scale respectively. The data are compared with the NLO QCD calculation ${ }^{3}$ for MRSD0 parton distributions and a renormalization scale choice of $\mu=E_{T} / 2$. The data and the predictions are in very good agreement over 9 orders of magnitude, with a small excess in the data at both low and high $E_{T}$. The renormalization scale dependence and the dependence on the choice of the parton distribution each amount to about a $15 \%$ variation in the prediction. Also shown in Fig. 1a) is the prediction for a contact term added to the LO QCD Lagrangian. ${ }^{4}$ Comparing the prediction with the data above $E_{T}>200 \mathrm{GeV}$, we calculate the $95 \%$ confidence level on the compositeness parameter to be $\Lambda_{c}>1450 \mathrm{GeV}$.

\section{The Two-Jet Differential Cross Section}

The two-jet differential cross section can be used to probe the parton distributions over a wide range of $x$ and to study the behavior of the cross section near the edges of kinematic phase space. For this analysis, only a subset $(\approx 40 \%)$ of the $1992-93$ data has been used. For each event, one jet is required to lie in the region $0.1<|\eta|<0.7$, whilst the second jet is required to have an $E_{T}>10 \mathrm{GeV}$, and to lie in the pseudorapidity slices $0.1-0.7,0.7-1.2,1.2-1.6,1.6-2.0$ and 2.0-3.0. Jet energies are corrected to a 

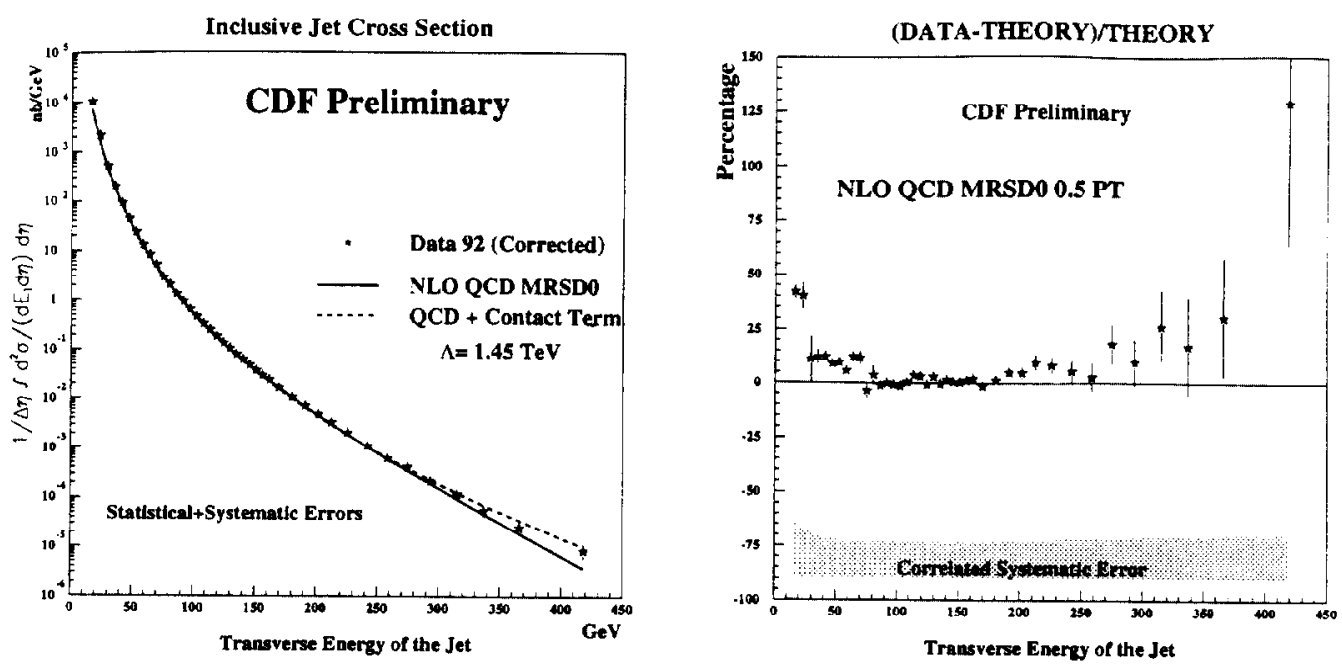

Fig. 1. Comparison of the inclusive jet cross section with NLO QCD on a) logarithmic and b) linear scales. The prediction from LO QCD+contact term is also shown.

common raw energy scale, defined by the central calorimeter. The cross sections have been corrected for detector effects due to energy resolution, but not for those due to $\eta$-resolution. The systematic uncertainty varies from about $15 \%$ to $30 \%$ on the cross section and from $1 \%$ to $35 \%$ on the ratio of cross sections.

In Fig. 2a) and b), we show the corrected cross sections and cross-section ratios (non-central over central) as a function of $E_{T}$ for the various slices of $\left|\eta_{2}\right|$. The data are compared to the LO QCD predictions using MT-LO parton distributions and a renormalization scale of $\mu=E_{T}$. LO QCD provides a reasonable description of the data except for the highest $E_{T}$ and highest $\left|\eta_{2}\right|$ regions. There, we begin to see the effects of approaching the edges of the kinematic phase space. A recent NLO QCD calculation $^{5}$ shows that there is a substantial correction to the LO prediction in this region, thereby bringing the theory into much better agreement with the data.

\section{The SS-OS Dijet Ratio}

The SS-OS dijet ratio is particularly sensitive to the gluon distribution at small $x$. The SS (OS) jet cross section is obtained by selecting events with two leading jets whose pseudorapidities have the same absolute values and the same (opposite) signs. The ratio of these cross sections has reduced experimental and theoretical errors. The small- $x$ sensitivity of the ratio is easily understood: At large $|\eta|$ and small $E_{T}$, the 

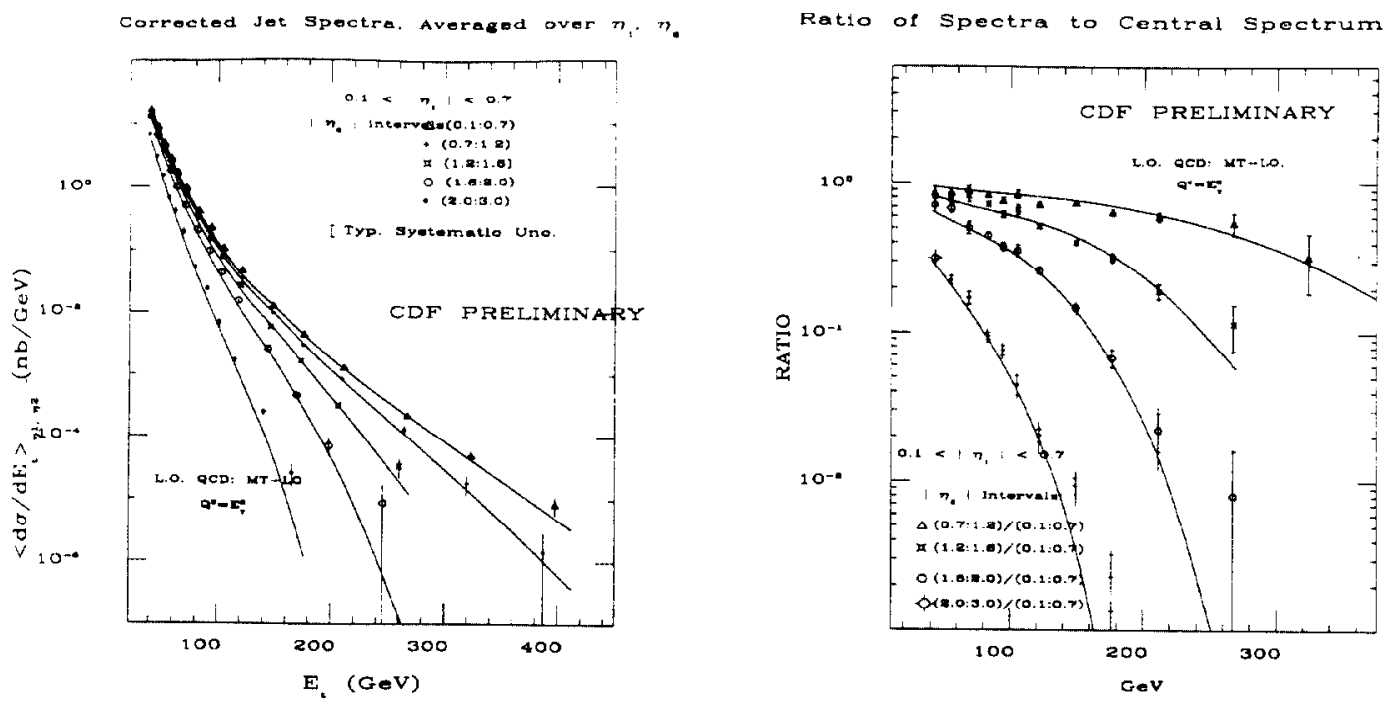

Fig. 2. The a) two-jet cross section and b) ratio of two-jet cross sections for various $\left|\eta_{2}\right|$ slices

SS jet cross section is dominated by gluon-quark scattering, with the quark having a very large $x$ and the gluon having a very small $x$. On the other hand, the OS jet cross section is sensitive to $x$ values where the parton distributions are relatively well known. The value of the ratio at large $\eta$ rises faster for a singular gluon distribution than a nonsingular one.

For this analysis, jet energies have been corrected to a common central energy scale. In order to remove some systematic biases, the azimuthal separation between the two leading jets is required to lie in the range $\pi-0.7-\pi+0.7$. In Fig. $3 \mathrm{a})-3 \mathrm{~d}$ ), we show the measured values of the ratio as a function of $\eta_{1}$ for the four $E_{T}$ regions analysed. They are compared with the predictions of LO QCD for the CTEQ2M and CTEQ2MS parton distribution, where the theoretical predictions have been smeared to take into account the detector effects. These effects, which become important as $|\eta|$ becomes large, are still under study. Overall, the data and the smeared predictions are in qualitative agreement in shape, with some evidence at low $E_{T}$ supporting the singular gluon hypothesis at small $x$. At higher $E_{T}$ 's, the ratio provides another test of QCD near the edges of kinematic phase space.

\section{References}

1. CDF Collaboration, F. Abe et al., Phys. Rev. D45 (1992) 1448 . 
2. CDF Collaboration, F. Abe et al., Phys. Rev. Lett. 70 (1992) 1376.

3. S. Ellis et al., Phys. Rev. Lett. 62 (1989) 2188, Phys. Rev. Lett. 64 (1990) 2121.

4. E. Eichten, K. Lane, and M. Peskin, Phys. Rev. Lett. 50 (1983) 811.

5. W.T. Giele et al., FERMILAB-PUB-94/070-T (1994).
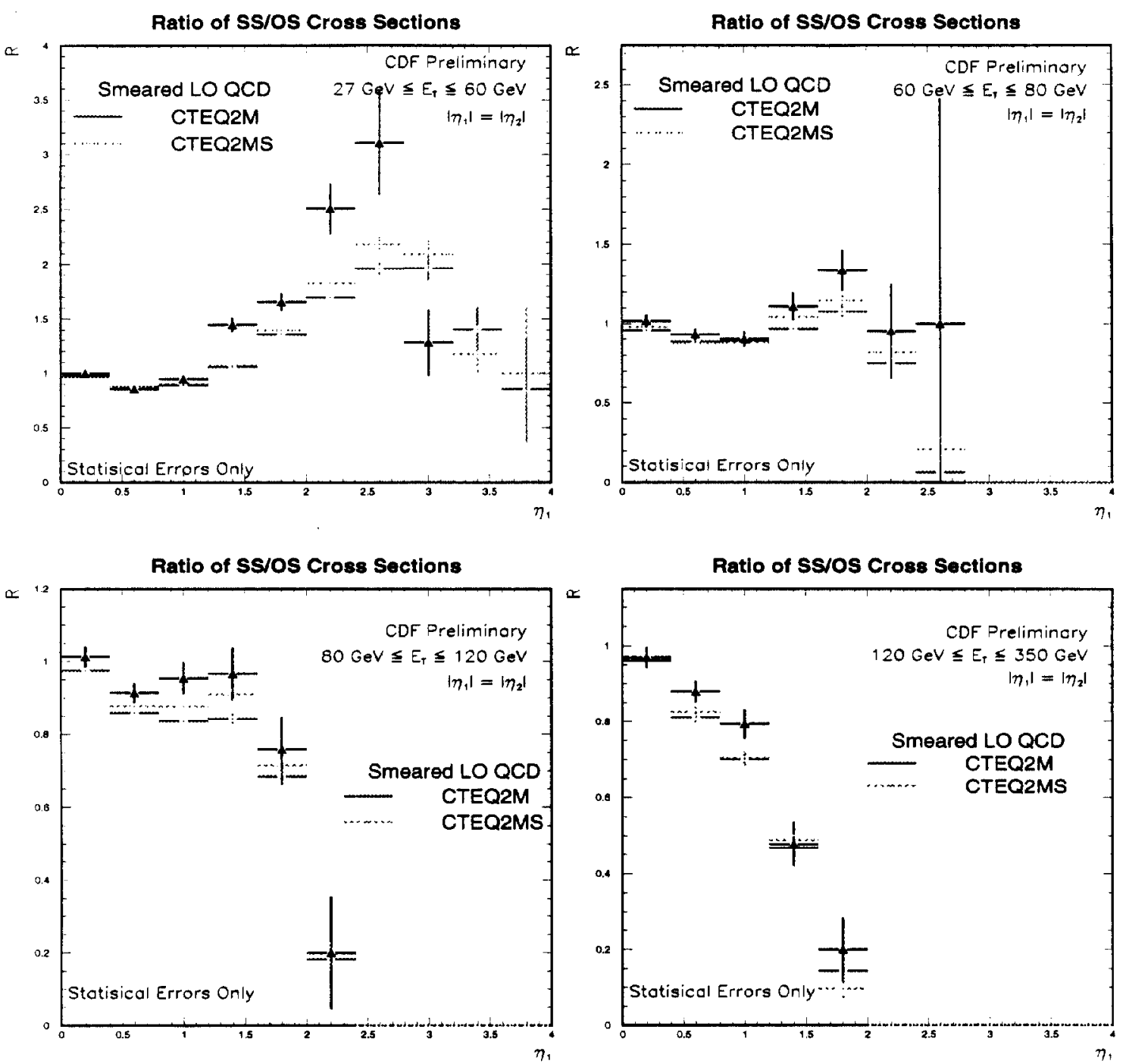

Fig. 3. The measured and simulated values of $R$ as a function of $\eta_{1}$ in the measured $E_{T}$ ranges a) 27-60 GeV, b) $60-80 \mathrm{GeV}$, c) $80-120 \mathrm{GeV}$, and d) 120-350 GeV. Data points are indicated by solid triangles. 